\title{
Polysomnographic Findings in Dementia With Lewy Bodies
}

\author{
Winnie C. Pao, MD, Bradley F. Boeve, MD, Tanis J. Ferman, PhD, Sioung-Chi Lin, MD, \\ Glenn E. Smith, PhD, David S. Knopman, MD, Neill R. Graff-Radford, MBChB, Ronald C. \\ Petersen, PhD, MD, Joseph E. Parisi, MD, Dennis W. Dickson, MD, and Michael H. Silber, \\ $\mathrm{MBCbB}$ \\ Departments of Neurology (Drs. Pao, Boeve, Knopman, Graff-Radford, Petersen, Parisi, Silber), \\ Psychology and Psychiatry (Drs Ferman and Smith), Center for Sleep Medicine (Drs. Pao, \\ Boeve, Lin, Silber), Laboratory Medicine and Pathology (Dr. Parisi), and Neuroscience \\ (Neuropathology Laboratory) (Dr. Dickson), Mayo Clinic College of Medicine; and Robert H. and \\ Clarice Smith and Abigail Van Buren Alzheimer's Disease Research Program of the Mayo \\ Foundation, Rochester, Minnesota, and Jacksonville, Florida
}

\section{Abstract}

Introduction-The clinical features of dementia with Lewy bodies (DLB) during wakefulness are well known. Other than REM sleep behavior disorder (RBD), only limited data exists on other sleep disturbances and disorders in DLB. We sought to characterize the polysomnographic (PSG) findings in a series of DLB patients with sleep-related complaints.

Methods-Retrospective study of patients with DLB who underwent clinical PSG at Mayo Clinic Rochester or Mayo Clinic Jacksonville over an almost 11 year span for evaluation of dream enactment behavior, excessive nocturnal movements, sleep apnea, hypersomnolence, or insomnia. The following variables were analyzed: respiratory disturbance index (RDI) in disordered breathing events/hour, periodic limb movement arousal index (PLMAI), arousals for no apparent reason (AFNAR), total arousal index (TAI), presence of REM sleep without atonia (RSWA), and percent sleep efficiency (SE).

Results-Data on 78 patients $(71 \mathrm{M}, 7 \mathrm{~F})$ were analyzed. The mean age was $71 \pm 8$ years. Seventy-five (96\%) patients had histories of recurrent dream enactment during sleep with $83 \%$ showing confirmation of RSWA +/- dream enactment during PSG. Mean RDI $=11.9 \pm 5.8$, PLMAI $=5.9 \pm 8.5$, AFNARI $=10.7 \pm 12.0$, and $\mathrm{TAI}=26.6 \pm 17.4$. SE was $<80 \%$ in $72 \%$ of the sample, $<70 \%$ in $49 \%$, and $<60 \%$ in $24 \%$. In patients who did not show evidence of significant disordered breathing ( 23 with $\mathrm{RDI}<5$ ), $62 \%$ of arousals were AFNARs. In those patients who had significant disordered breathing (55 with RDI 25 ), 36\% of arousals were AFNARs. Six patients underwent evaluations with PSG plus MSLT. Two patients had mean initial sleep latencies less than five minutes, and both had RDI $<5$. No patient had any sleep onset rapid eye movement periods. Nineteen patients have undergone neuropathologic examination, and 18 have had limbicor neocortical-predominant Lewy body pathology. One had progressive supranuclear palsy, but no REM sleep was recorded in prior PSG.

Conclusions-In patients with DLB and sleep-related complaints, several sleep disturbances in addition to RBD are frequently present. In this sample, about three quarters had a significant number of arousals not accounted for by a movement or breathing disturbance, and the primary sleep disorders do not appear to entirely account for the poor sleep efficiency in DLB, especially in those without a significant breathing disorder. Further studies are warranted to better understand

Correspondence: Bradley F. Boeve, M.D., Mayo Clinic, 200 First Street SW, Rochester, Minnesota 55905, Phone: (507) 538-1038, Fax: (507) 538-6012, bboeve@ mayo.edu. 
the relationship between disturbed sleep, arousal and DLB; such characterization may provide insights into potential avenues of treatment of symptoms which could impact quality of life.

\section{Keywords}

Sleep disorders; REM sleep behavior disorder; dementia with Lewy bodies; synucleinopathy

\section{Introduction}

Dementia with Lewy bodies (DLB) refers to the constellation of features that are typically associated with limbic or neocortical Lewy body disease (1). DLB is now considered the second most common cause of neurodegenerative dementia after Alzheimer's disease (2). The revised Consensus criteria (1) for the clinical diagnosis of DLB requires the presence of dementia plus the following core features: fluctuations in cognition or arousal, recurrent and fully formed visual hallucinations, and spontaneous parkinsonism. Two of these features are required for the diagnosis of probable DLB, and one for the diagnosis of possible DLB. A diagnosis of probable DLB may also be made with the presence of 1 core feature and one of the following suggestive features: REM sleep behavior disorder, severe neuroleptic sensitivity or low dopamine transporter uptake in the basal ganglia on functional imaging. The importance of the diagnosis is underscored by the often positive response to cholinesterase inhibitors and the exquisite sensitivity often demonstrated with anticholinergics and neuroleptics with D2 antagonism (1).

REM sleep behavior disorder (RBD) is a parasomnia in which patients appear to "act out their dreams." Ample data now suggests RBD occurs frequently in the synucleinopathies [e.g., DLB, Parkinson's disease (PD) with and without dementia, and multiple system atrophy (MSA)] and rarely in the tauopathies $(3,4)$. Some have argued that RBD should be considered a core feature of the disorder $(5,6)$. Our recent data has also shown that among those who have mild cognitive impairment associated with RBD who are followed longitudinally, almost all subsequently develop the other core features of DLB and all have underlying Lewy body disease found at autopsy (7). Hence, RBD is clearly frequent in DLB and usually precedes the onset of the other core features of the disorder by many years.

There is little data on other common sleep disorders in those with DLB, such as upper airway resistance syndrome (UARS), obstructive sleep apnea/hypopnea syndrome (OSAHS), central sleep apnea syndrome (CSA), and periodic limb movements (PLMs) of sleep. Furthermore, to what degree these and other disorders cause sleep fragmentation, reduced sleep efficiency, insomnia, or hypersomnia in DLB patients is not known. These sleep disorders are relatively common in the neurologically normal elderly, and untreated sleep disorders clearly can affect cognition, mood, daily functional status, and quality of life. The majority of sleep disorders are treatable, and hence appropriate therapy could improve nocturnal sleep continuity, reduce daytime hypersomnolence, and improve daytime functioning and quality of life for DLB patients and their bed partners/caregivers. Sleep disturbances are a major contributor to caregiver decision-making in institutionalizing their loved ones who have dementia, which underscores the importance of recognizing, diagnosing, and treating the multitude of sleep disorders in this patient population. No systematic study has ever attempted to address the frequency of sleep disturbances in DLB.

In this study, we sought to characterize the polysomnographic (PSG) findings in a series of DLB patients who presented with sleep-related complaints to study the scope and relative frequency of sleep disturbances in this population. 


\section{Design/Methods}

\section{Case ascertainment}

We identified all DLB patients evaluated by one or more of the authors who underwent sleep medicine consultations and PSG at Mayo Clinic Rochester and Mayo Clinic Jacksonville between $1 / 92$ and 10/02.

\section{Inclusion criteria}

All subjects met criteria for clinically possible or probable DLB (1). All patients and/or bed partners had sleep-related complaints warranting clinical sleep evaluations and PSGs, which included one or more of the following features: recurrent dream enactment behavior, excessive nocturnal movements, disruptive snoring with observed apnea, excessive daytime somnolence, or insomnia.

\section{Data collection}

Polysomnographic recording and scoring were performed according to established guidelines (8). All PSG studies involved continuous video synchronized to standard PSG monitoring using the following montage: two electro-oculogram (EOG) derivations, three EEG derivations (Fz-Cz, Cz-Oz, C4-A1), electrocardiogram (ECG), chin and at least two limb surface electromyograms (EMG), oronasal airflow, sonogram, oxyhemoglobin saturation, and chest and abdomen inductance plethysmography. Scoring of sleep stages followed standard guidelines (12), and each PSG was reviewed by a sleep medicine clinician certified by the American Board of Sleep Medicine (ABSM). Since all PSGs were performed during the 1992-2002 period which preceded the updated 2007 guidelines for defining and measuring sleep-related events (9), the definitions for sleep-related events which were being used over 1992-2002 time period (10) are the definitions used in this study. The finding of REM sleep without atonia (RSWA) was considered present if muscle tone during REM sleep was considered unequivocally abnormally increased by an ABSMcertified sleep clinician and if no epileptiform discharges were noted on the record (8). An arousal was defined as an abrupt shift of EEG frequency including alpha, theta or frequency higher than $16 \mathrm{~Hz}$ but not sleep spindles after at least 10 seconds of stable sleep, that lasted at least 3 seconds during any sleep stage but not long enough to be classified as awake. An arousal during REM sleep was scored only if it was accompanied by increased amplitude of submental EMG. A disordered breathing event (DBE) was defined as an obstructive apnea, central apnea, mixed apnea, hyponea episode, or respiratory effort related arousal (RERA). The disordered breathing arousal index (DBAI) was defined as the average number of arousals per hour associated with a disordered breathing event. For an obstructive event, there was a 10 seconds or longer period with clear amplitude decrease in breathing from baseline associated with over $3 \%$ oxygen desaturation or an arousal from the obstructive events. In a central event, there was a reduction or absence of breathing and respiratory effort that lasted 10 seconds or longer, with associated reduced airflow and esophageal pressure. For a hypopnea, there was a reduction of airflow and reduction of thoracic and/or abnormal movement that lead to an arousal. RERA was considered present when a respiratory effort such as a snort was associated with an arousal. The respiratory disturbance index (RDI) represents the sum of disordered breathing events related to obstructive apneas, central apneas, mixed apneas, hypopneas, and respiratory effort related changes averaged over the total sleep time, which represents a value of this sum per hour. A periodic limb movement (PLM) was defined as periodic contraction of lower legs either unilateral or bilateral, with a series of 4 consecutive movements separated by 4 to 90 seconds, each movement lasted between 0.5 to 5 seconds, and was not associated with respiratory events. The mean number of PLMs per hour associated with arousals was considered the PLMAI. An arousal for no apparent reason (AFNAR) was defined as an arousal not related to 
disordered breathing or movements, and the AFNAR index (AFNARI) represents the number of AFNARs per hour averaged over total sleep time. Sleep efficiency was defined as the total sleep time divided by the total time in bed multiplied by $100 \%$.

We routinely perform split-night studies for those patients who have clinically significant sleep disordered breathing, meaning that those who have an RDI of 5 or more per hour are routinely initiated in continuous positive airway pressure (CPAP) after 3 or 4 hours of the study. For such patients, the initial hours of observational assessment of sleep is considered the diagnostic portion of the study, and the subsequent portion in which the patients are administered CPAP therapy is considered the therapeutic portion of the study. For patients who had full-night PSGs (i.e., had no significant disordered breathing events and therefore CPAP therapy was not administered), data over the entire study were analyzed. For those patients who had split-night studies (i.e., had significant disordered breathing such that CPAP therapy was initiated half-way through the night), only data from the initial diagnostic portion of the study when the patients were not on CPAP were analyzed.

For patients who underwent evaluations for hypersomnolence, all psychoactive medications were suggested to be discontinued for at least two weeks in preparation for a PSG/multiple sleep latency test (MSLT), and at least seven hours of total sleep time was attempted on the PSG immediately prior to the MSLT. Medications which were preferentially discontinued included sedative/hypnotics, clonazepam, antidepressants, and psychostimulants. For ethical reasons, cholinesterase inhibitors tended to be maintained due to the rare but well-known phenomenon of marked clinical worsening with cholinesterase inhibitor withdrawal that does not reverse upon reinstitution of the drug. Donepezil was always administered once daily in the morning, and the second daily dose of rivastigmine or galantamine were administered no later than suppertime. Note that the extended release preparations of transdermal rivastigmine and extended release galantamine became available over more recent years, and were not yet available at during the observation period of this analysis $(1 / 92-10 / 02)$. Also for ethical reasons, if visual hallucinations, delusions, agitation, etc were viewed too severe to justify discontinuation of atypical neuroleptics, which in our patient population involves quetiapine almost exclusively, this agent was maintained.

\section{Data analysis}

The following variables were analyzed on the PSGs: presence of REM sleep without atonia (RSWA), respiratory disturbance index (RDI), periodic limb movement arousal index (PLMAI), arousals for no apparent reason index (AFNARI), total arousal index (TAI), and percent sleep efficiency (SE). Total arousal index is further subdivided into percentages from disordered breathing, movement, or no apparent reason. The diagnosis of upper airway resistance syndrome (UARS) was made for patients who had five or more respiratory effort related arousals per hour. The diagnosis of obstructive sleep apnea/hypopnea syndrome (OSHAS) was made for patients who had five or more obstructive hypopneas or apneas with arousals per hour. The diagnosis of central sleep apnea syndrome (CSAS) was made for patients who had five or more central hypopneas or apneas with arousals per hour. For those who underwent MSLT, the mean initial sleep latency (ISL) and number of sleep onset rapid eye movement periods (SOREMPs) were analyzed. Values on the mean ISL were viewed as follows: $>10$ minutes $=$ normal,$<5$ minutes $=$ severe hypersomnolence, $5-10$ minutes $=$ borderline hypersomnolence (additional clinical data is required to interpret the MSLT data and decide on therapy). The presence of 2 or more SOREMPs was considered compatible with the diagnosis of narcolepsy in the appropriate clinical setting. 


\section{Informed consent and IRB approval}

All patients (or their proxies) provide written consent prior to undergoing PSGs +/- MSLTs, and spouses/caregivers are routinely requested to spend the night in the room of the patient's sleep study to help orient the patient and provide comfort if disorientation and anxiety occur during the study. Most of the DLB subjects were enrolled in one of the aging and dementia studies at Mayo Clinic - the Neuropsychology of DLB Study (AG015866), the Mayo Alzheimer's Disease Research Center (AG016574), or the Mayo Clinic Study on Aging (AG006786), which are Mayo Foundation Institutional Review Board (IRB)-approved protocols. This specific analysis was also reviewed and approved by the Mayo IRB.

\section{Results}

Data on 78 patients ( $71 \mathrm{M}, 7 \mathrm{~F})$ were analyzed (Table 1). The mean age was $71 \pm 8$ years. Severity of dementia ranged from mild to moderate (mean total Mattis Dementia Rating Scale Score $=115 \pm 18$ ). Recurrent dream enactment behavior during sleep was reported to occur in 75 (96\%) of the DLB subjects, and 65 (83\%) of them had RSWA during PSG and thus confirmed RBD in these patients. Thirteen $(17 \%)$ of the subjects did not attain any REM sleep, and hence RSWA could not be assessed. One patient without a history of RBD had RSWA. OSAHS criteria were met in 43 (55\%) subjects. Fourteen (18\%) had central hypopneas or apneas present, and one met criteria for the diagnosis of CSAS.

The mean values on other PSG data were as follows: Sleep efficiency $<80 \%$ was found in $72 \%$ of the sample. Half the sample $(49 \%)$ had sleep efficiency $<70 \%$, and $26 \%$ of the sample had sleep efficiency $<60 \%$ (Table 1 ). Sleep efficiency did not show any statistically significant correlation with age, dementia severity, BMI or PLM in our study. Other mean ( \pm $\mathrm{SD}$ ) values were: $\mathrm{RDI}=11.9 \pm 15.8, \mathrm{PLMAI}=5.9 \pm 8.5$, AFNARI $=10.7 \pm 12.0$, and TAI $=26.6 \pm 17.4$. Forty-seven patients $(60 \%)$ patients had DBAI $\geq 5,35(45 \%)$ patients had PLMAI $\geq 5,59(76 \%)$ had AFNARI $\geq 5$, and all $78(100 \%)$ had TAI $\geq 5$.

Twenty-three patients who had no evidence of clinically significant disordered breathing (RDI<5) underwent full-night studies (Table 2); sleep efficiency was $72.3 \%$ (SD 13.6). The total arousal index was 16.6 (SD 8.3), in which 61.9\% (SD 22.3) was attributed to AFNAR, with $83 \%$ having an AFNARI $\geq 5$ (Tables 3 and 4). The remaining 55 patients with presence of disordered breathing, the total arousal index was 30.9 (SD 18.6), and 44.5\% (SD 26.4) was due to breathing disorder, while 26.0\% (SD 20.7) from AFNAR (Table 4).

Six patients underwent evaluations with PSG plus MSLT. Two patients had mean initial sleep latencies on the MSLT less than five minutes, and both had DBAI $<5$. No patient had any SOREMPs. Both with abnormal MSLT findings were diagnosed with a form of "idiopathic hypersomnia" which in hindsight may be better viewed as hypersomnia associated with an underlying neurodegenerative disorder, and both were treated with psychostimulants and benefited from such treatment.

Nineteen of these subjects have died and undergone neuropathologic examination (Table 5) -4 had limbic- and 14 had neocortical-predominant Lewy body pathology (1), while one with progressive supranuclear palsy (PSP). The patient with PSP had no REM sleep recorded on PSG, though a history of RBD was suspected. RSWA was detected in all but 4 neocortical-predominant Lewy body (LB) pathology patients. One (with neocorticalpredominant type) out of these 19 patients was evaluated with MSLT, with a mean initial sleep latency being less than 5 minutes, and no SOREM recorded. The limbic- and neocortical predominant LB pathology patients had similar sleep efficiency and AFNAR profile, but the neocortical-predominant LB pathology patients showed a higher trend in RDI and PLMAI than the limbic-predominant LB pathology patients. The PSP patient had a 
much better sleep efficiency in the study, and relatively lower AFNARI, and minimal PLMAI.

\section{Discussion}

In this large cohort of patients with DLB and sleep-related complaints, several sleep disturbances in addition to RBD were frequently present. To summarize, most had historical and electrophysiologic evidence of RBD, most had reduced sleep efficiency, most had some degree of sleep-disordered breathing, most had some degree of periodic limb movements during sleep, most had some degree of arousals for no apparent reason, and a minority has historical and electrophysiologic evidence of excessive daytime somnolence, although findings consistent with narcolepsy were absent in this cohort

In $70 \%$ of the patients, sleep efficiency was below $80 \%$, with arousals attributable to disordered breathing, periodic limb movements during sleep, and spontaneous arousals. Sleep efficiency was not associated with age, dementia severity or body mass index. Despite the findings that $60 \%$ of the sample had an RDI $\geq 5$, only $36 \%$ of the sample had an RDI $\geq$ 10 , indicating that moderate or severe sleep apnea does not entirely account for the poor sleep efficiency. Thus, when sleep apnea is present in this cohort, it tends to be mild and may contribute to poor sleep efficiency, but may not be solely responsible for it. When the sample includes only those with a RDI $<5$, spontaneous arousals appear to be a predominant source of sleep disruption (83\%), and periodic limb movements are present in less than half $(43 \%)$. Arousals that are not accounted for by breathing or movement disturbance are common, though a subset of patients do experience movement-related arousals associated with PLMs. REM sleep without atonia, the electrophysiologic substrate of REM sleep behavior disorder, was present in the vast majority of patients (96\%), but did not solely account for poor sleep efficiency.

Sleep clinicians routinely search for historical clues and examination findings that make one suspicious for disordered breathing (loud disruptive snoring, observed snorts, gasps, and apneic pauses while sleeping, obesity, large collar size, crowded oropharynx); and for features suspicious of PLMs fragmenting sleep (such as RLS or leg jerks as reported by the patient or bedpartner, history of GI blood loss or anemia, clinical evidence of a peripheral neuropathy or radiculopathy). Yet despite comprehensive sleep histories and general and neurologic examinations, we continue to be struck by the paucity of clues for disordered breathing and PLMs that can be elicited during the interview and examination, while so many of these DLB patients have the primary issues of daytime hypersomnia, insomnia, or dream enactment behavior warranting PSG. Our findings presented herein and continued clinical experience in evaluating and following patients with DLB have indicated that almost every DLB patient has one or more potentially treatable sleep disorders, even in the absence of obvious historical features. We now have a low threshold for performing clinical PSGs in DLB subjects who exhibit features of daytime hypersomnia, nocturnal insomnia, dream enactment behavior, or some combination of these.

UARS, OSAHS, and PLMs during sleep were present in a proportion of these patients. About three quarters of the sample, however, had a significant number of arousals not accounted for by a movement or breathing disturbance (see Table 2). In a study by Unruh et al (12) which evaluated 5407 community dwelling adults that participated in the Sleep Heart Health Study that had undergone unattended home polysomnograms, 1719 were 60 years or older. The sleep efficiency for males is $80.2 \%$ (SD 10.9) for 60-69 year olds; $78.4 \%$ (SD 10.4 ) for $70-79$ year olds; and $73.6 \%$ (SD 12.3) for those 80 years or older. One would expect in our patient sample the sleep efficiency will be lower as the patients and/or caregivers all complained of some type of sleep disturbances. Sleep efficiency in our group 
was $<70 \%$ in half of the subjects. Yet the high number of arousals for no apparent reason, especially in the group that does not show evidence of breathing disorder (see Tables 2 and 3 ), indicates that primary sleep disorders such as UARS, OSA, and PLMs do not entirely account for the poor sleep efficiency in DLB. These findings underscore the need to better understand the pathophysiologic mechanisms underlying arousals for no apparent reason. Instability in the wakefulness/sleep flip-flop switch could be one cause, and considering the sleep-wake problems in those with Parkinson's disease and recent evidence of hypocretin-1 dysfunction in PD $(13,14)$ - another phenotype of underlying LBD - one could hypothesize that hypocretin-1 dysfunction contributed to some of the complex pathophysiology of sleep fragmentation, drowsiness and cognitive fluctuations in DLB. The high degree of accuracy of the DLB syndrome predicting underlying LBD pathology (Table 5) underscores the biologic significance of our findings

Of the 19 subjects in the study that have died and undergone neuropathologic examination, 4 had limbic-predominant, and 14 had neocortical-predominant Lewy body pathology. The two groups have similar sleep efficiency and AFNARI, and a higher trend in RDI and PLMAI is noticed in the neocortical-predominant patients. Though there was a history suggestive of RBD in our PSP patient, we were unable to confirm RBD as no REM sleep was recorded in PSG. Interestingly, our PSP patient had a much better sleep efficiency than the DLB patients, with minimal PLMAI, and lower AFNARI. It will be interesting to see if AFNARs are also frequent in other neurodegenerative dementia with daytime somnolence, or if it is more specific to patients with DLB. Given that our autopsied study patient number with PSG is limited, larger groups of patients and with different dementia subtypes will be needed to verify our findings.

We acknowledge the limitations in this study. Our findings should not be interpreted as reflecting the actual prevalence of sleep disturbances and disorders in DLB, since our patients underwent PSGs for clinical purposes and are hence biased toward more sleep morbidity. It also should not be considered as the full scope of sleep disorders in DLB, since only six patients underwent MSLTs, and more might have met criteria for the diagnosis of narcolepsy or idiopathic hypersomnia had they undergone full PSG/MSLT evaluations. Performing and scoring PSGs in those with DLB are challenging - some refuse to undergo PSG for a variety of reasons, some become combative/uncooperative during the PSG which necessitates early discontinuation of the study, many have slowing on their background EEG and some have features of status dissociatus which make scoring the PSG itself quite difficult.

Yet these findings suggest that further prospective studies are warranted to better understand the relationship between sleep disturbances, cognition, arousal, fluctuations and DLB. Furthermore, successful diagnosis and treatment of sleep disorders may promote daytime functioning and quality of life for patients and their caregivers, and delay the need for institutionalization. Even purely from the financial perspective, the expenses associated with a sleep medicine consultation, polysomnogram, medication(s), or continuous positive airway pressure (CPAP) would equate to 1-2 months worth of skilled nursing care expenses, suggesting that a delay in nursing home placement from successful treatment of one or more sleep disorders could reduce health care costs for some families by thousands or tens of thousands of dollars. Health care expenditures associated with successful treatment of sleep disorders in DLB patients (and dementia patients in general) is certainly worthy of future research as well.

\section{Acknowledgments}

Supported by NIA grants AG015866, AG006786, AG016574, and the Mangurian Foundation 


\section{References}

1. McKeith IG, Dickson DW, Lowe J, et al. Diagnosis and management of dementia with Lewy bodies: third report of the DLB Consortium. Neurology. 2005; 65:1863-1872. [PubMed: 16237129]

2. McKeith IG, Galasko D, Kosaka K, et al. Consensus guidelines for the clinical and pathologic diagnosis of dementia with Lewy bodies (DLB): report of the consortium on DLB international workshop. Neurology. 1996; 47:1113-1124. [PubMed: 8909416]

3. Boeve BF, Silber MH, Ferman TJ, Lucas JA, Parisi JE. Association of REM sleep behavior disorder and neurodegenerative disease may reflect an underlying synucleinopathy. Mov Disord. 2001; 16:622-630. [PubMed: 11481685]

4. Boeve BF, Silber MH, Parisi JE, et al. Synucleinopathy pathology and REM sleep behavior disorder plus dementia or parkinsonism. Neurology. 2003; 61:40-45. [PubMed: 12847154]

5. Ferman TJ, Boeve BF, Smith GE, et al. Dementia with Lewy bodies may present as dementia and REM sleep behavior disorder without parkinsonism or hallucinations. J Int Neuropsychol Soc. 2002; 8:907-914. [PubMed: 12405541]

6. Turner RS. Idiopathic rapid eye movement sleep behavior disorder is a harbinger of dementia with Lewy bodies. J Geriatr Psychiatry Neurol. 2002; 15:195-199. [PubMed: 12489915]

7. Molano J, Boeve B, Ferman T, et al. Mild cognitive impairment associated with limbic and neocortical lewy body disease: a clinicopathological study. Brain. 2010; 133:540-556. [PubMed: 19889717]

8. Rechtschaffen, A.; Kales, A. A Manual of standardized terminology, techniques and scoring system for sleep stages of human subjects. Public Health Service, U S Government Printing Office; 1968.

9. Iber, C.; Ancoli-Israel, S.; Chesson, A.; Quan, S. for the American Academy of Sleep Medicine. The AASM manual for the scoring of sleep and associated events: rules, terminology and technical specifications. 1. Westchester: IL: American Academy of Sleep Medicine; 2007.

10. The Report of an American Academy of Sleep Medicine Task Force. Sleep-related breathing disorders in adults: recommendations for syndrome definition and measurement techniques in clinical research. Sleep. 1999; 22:667-89. [PubMed: 10450601]

11. Lapierre O, Montplaisir J. Polysomnographic features of REM sleep behavior disorder: development of a scoring method. Neurology. 1992; 42:1371-1374. [PubMed: 1620348]

12. Unruh ML, Redline S, An MW, et al. Subjective and objective sleep quality and aging in the sleep heart health study. J Am Geriatr Soc. 2008; 56:1218-1227. [PubMed: 18482295]

13. Thannickal TC, Lai YY, Siegel JM. Hypocretin (orexin) cell loss in Parkinson's disease. Brain. 2007; 130:1586-95. [PubMed: 17491094]

14. Fronczek R, Overeem S, Lee SY, et al. Hypocretin (orexin) loss in Parkinson's disease. Brain. 2007; 130:1577-85. [PubMed: 17470494] 
Table 1

Characteristics and PSG analysis of 78 clinically diagnosed DLB patients with sleep-related complaints

\begin{tabular}{|c|c|c|}
\hline & mean & SD \\
\hline age & 71.7 & 7.7 \\
\hline $\mathrm{n}$ & 78 & male $=71$ \\
\hline GLDS $(n=71)$ & 3.7 & 0.9 \\
\hline DRS (n=57) & 115 & 18 \\
\hline \multirow[t]{2}{*}{ BMI (n=48) } & 26.7 & 4.6 \\
\hline & $\mathbf{n}$ & $\%$ \\
\hline VH & 37 & 47 \\
\hline Parkinsonism & 50 & 64 \\
\hline RBD & 65 & 83 \\
\hline RSWA & 66 & 85 \\
\hline RDI $\geq 5$ & 47 & 60 \\
\hline $\mathrm{RDI} \geq 10$ & 28 & 36 \\
\hline PLMAI $\geq 5$ & 35 & 45 \\
\hline AFNARI $\geq 5$ & 59 & 76 \\
\hline TAI $\geq 5$ & 78 & 100 \\
\hline $\mathrm{SE}<80 \%$ & 56 & 72 \\
\hline $\mathrm{SE}<70 \%$ & 38 & 49 \\
\hline \multirow[t]{2}{*}{$\mathrm{SE}<60 \%$} & 19 & 24 \\
\hline & mean & SD \\
\hline RDI & 11.9 & 15.8 \\
\hline PLMAI & 5.9 & 8.5 \\
\hline AFNARI & 10.7 & 12 \\
\hline TAI & 26.6 & 17.4 \\
\hline
\end{tabular}

Abbreviations: AFNARI=arousal for no apparent reason index; BMI=body mass index; $\mathrm{DEB}=\mathrm{dream}$ enactment behavior; $\mathrm{DLB}=\mathrm{dementia}$ with Lewy bodies; DRS=Dementia Rating Scale (maximum score $=144$, values in the 100-120 range are generally considered mild to moderate dementia); GLDS=Global Deterioration Scale (3=mild dementia, 4=moderate dementia, 5=moderately severe dementia); PLMAI=periodic limb movement arousal index; $\mathrm{PSG}=$ polysomnogram; RDI=respiratory disturbance index; RSWA=REM sleep without atonia; $\mathrm{SE}=$ sleep efficiency; TAI=total arousal index. 
Table 2

Characteristics and PSG analysis of 23 clinically diagnosed DLB patients with RDI $<5$

\begin{tabular}{lcc}
\hline & mean & SD \\
\cline { 2 - 3 } age & 69.6 & 8.4 \\
$\mathrm{n}$ & 23 & male $=20$ \\
GLDS (n=19) & 3.6 & 0.9 \\
DRS (n=12) & 122.5 & 17.9 \\
BMI $(\mathrm{n}=12)$ & 23.6 & 3.5 \\
& $\mathbf{n}$ & $\%$ \\
\cline { 2 - 3 } History of recurrent DEB & 23 & 100 \\
RSWA & 21 & 91 \\
PLMAI $\geq 5$ & 10 & 43 \\
AFNARI $\geq 5$ & 19 & 83 \\
TAI $\geq 5$ & 23 & 100 \\
SE $<80 \%$ & 16 & 70 \\
SE $<70 \%$ & 9 & 39 \\
SE $<60 \%$ & 4 & 17 \\
\hline
\end{tabular}

Abbreviations: AFNARI=arousal for no apparent reason index; $\mathrm{BMI}=$ body mass index; $\mathrm{DEB}=\mathrm{dream}$ enactment behavior; $\mathrm{DLB}=\mathrm{dementia}$ with Lewy bodies; DRS=Dementia Rating Scale (maximum score=144, values in the 100-120 range are generally considered mild to moderate dementia); GLDS=Global Deterioration Scale (3=mild dementia, 4=moderate dementia, 5=moderately severe dementia); PLMAI=periodic limb movement arousal index; PSG=polysomnogram; RDI=respiratory disturbance index; RSWA=REM sleep without atonia; SE=sleep efficiency; $\mathrm{TAI}=$ total arousal index. 


\section{Table 3}

PSG characterization of sleep and arousals in 23 patients with probable DLB and RDI $<5$

\begin{tabular}{lcc}
\hline & mean & SD \\
\cline { 2 - 3 } & & \\
Sleep efficiency $(\%)$ & 72.3 & 13.6 \\
Total arousal index (TAI) & 16.6 & 8.3 \\
Arousals for no apparent reason (\% of TAI) & 61.9 & 22.3 \\
Disordered breathing (\% of TAI) & 12.8 & 12.9 \\
Periodic limb movements (\% of TAI) & 25.2 & 23.6 \\
\hline
\end{tabular}

Abbreviations: $\mathrm{DLB}=$ dementia with Lewy bodies; $\mathrm{PSG}=$ polysomnogram; RDI=respiratory disturbance index; $\mathrm{SD}=$ standard deviation; TAI=total arousal index. 


\section{Table 4}

PSG characterization of sleep and arousals in 55 patients with probable DLB and RDI $\geq 5$

\begin{tabular}{lll}
\hline & mean & SD \\
\cline { 2 - 3 } Sleep efficiency (\%) & 67.8 & 18.2 \\
Total arousal index (TAI) & 30.9 & 18.6 \\
Arousals for no apparent reason (\% of TAI) & 36.5 & 23.5 \\
Disordered breathing (\% of TAI) & 44.5 & 26.4 \\
Periodic limb movements (\% of TAI) & 19.4 & 18.7 \\
\hline
\end{tabular}

Abbreviations: DLB=dementia with Lewy bodies; $\mathrm{PSG}=$ polysomnogram; RDI=respiratory disturbance index; $\mathrm{SD}=$ standard deviation; TAI=total arousal index. 


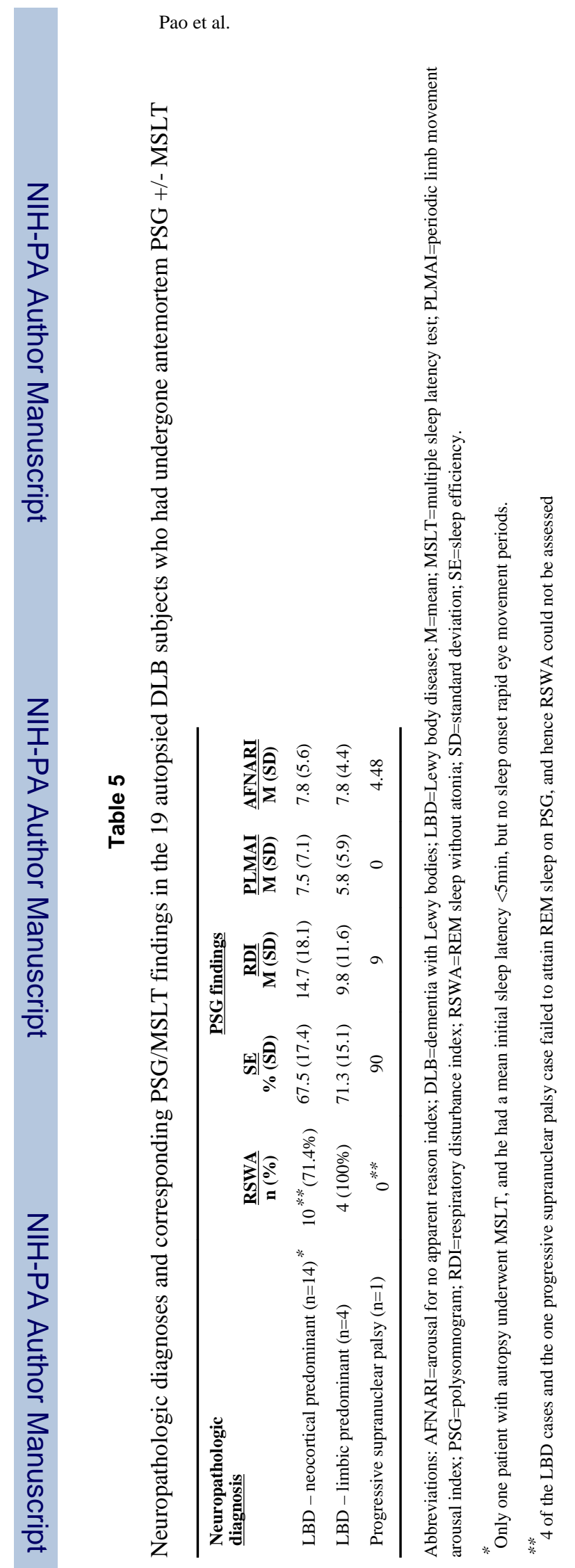

Neurologist. Author manuscript; available in PMC 2014 January 01. 